\title{
Determinação do trajeto do canal mandibular por meio de tomografia computadorizada em dez mandíbulas de cadáveres de cães mesaticefálicos
}

\author{
Mandibular canal course determination by means of computerized tomography in ten \\ mandibles of mesaticephalic dogs cadavers
}

\author{
Lenin Arturo VILLAMIZAR Martinez ${ }^{1}$; Marco Antonio GIOSO ${ }^{1}$; Cristian Marcelo Villegas LOBOS ${ }^{2}$; \\ Ana Carolina Brandão de Campos Fonseca PINTO'
}

${ }^{1}$ Departamento de Cirurgia da Faculdade de Medicina Veterinária e Zootecnia da Universidade de São Paulo, São Paulo-SP ${ }^{2}$ Instituto de Matemática e Estatística da Universidade de São Paulo, São Paulo-SP

\begin{abstract}
Resumo
Sabe-se que durante qualquer procedimento cirúrgico na região da mandíbula no homem, um dano iatrogênico ao feixe vasculonervoso que percorre o canal mandibular $(\mathrm{CM})$ poderia causar desde parestesia até dor constante. Na odontologia veterinária, diferentes procedimentos cirúrgicos são realizados no tecido ósseo adjacente ao CM, o que implica no conhecimento acurado da localização do mesmo. O objetivo desta pesquisa foi determinar por meio da Tomografia Computadorizada (TC) o trajeto do CM em relação às faces da mandíbula: lingual, vestibular, ventral e crista alveolar em dez mandíbulas de cadáveres de cães mesaticefálicos. Os tomogramas foram realizados no plano transversal, tomando como referência para cada corte o forame mandibular, as raízes dentárias dos dentes molares, pré-molares e forame mentoniano médio. No tomógrafo foram realizadas medidas desde o CM até as diferentes faces. Conclui-se que a partir do dente 3o molar, no sentido rostral, o CM aumenta gradualmente a distância em relação à crista alveolar, alcançando a sua máxima profundidade na região dente $1^{\circ}$ molar. Em relação às faces vestibular e lingual, o CM apresentou-se lingualizado na região dos dentes molares. O CM continua rostralmente ocupando a região ventral do corpo da mandíbula, mantendo uma distância similar entre a superfície vestibular e lingual. Já na região do dente $3^{\circ}$ pré-molar o canal mandibular aumenta ligeiramente a sua distância com relação à face ventral e lingual da mandíbula, antes do seu fim no forame mentoniano medial na face vestibular da mandíbula.
\end{abstract}

Palavras-chave: Canal mandibular. Tomografia computadorizada. Odontologia veterinária. Cães braquicefálicos.

\begin{abstract}
As it is known, during any surgical procedure in the human mandible, an iatrogenic damage to the neurovascular bundle that runs along the mandibular canal (MC) could cause from paresthesia to constant pain. In veterinary dentistry, different surgical procedures are performed on the bone tissue adjacent to the $\mathrm{MC}$, which implies accurate knowledge of its localization. The purpose of this study was to determine by means of computerized tomography (CT) the path of the MC in relation with: lingual surface, vestibular surface, alveolar crest, and ventral mandible surface in ten mesaticephalic dogs. The slices were performed in transverse plane using as reference the mandibular foramen, the medial mental foramen and the tooth roots of molars and premolars; several measures among the $\mathrm{MC}$ and the mandibular faces were performed. The conclusion of this study was that from the $3 \mathrm{rd}$ molar tooth (following in rostral direction), the MC gradually increases its distance from the alveolar crest, reaching its maximum depth in the 1st molar tooth area. In the molar area, the MC was located nearly of the mandibular lingual surface. The CM continues rostrally occupying the ventral region of the mandible body keeping a similar distance between the buccal and lingual surface. Then in the $3^{\text {rd }}$ premolar area the MC increases slightly its distance from the ventral aspect of the mandible, before its end in the medial mental foramen on the face of the mandible.
\end{abstract}

Keywords: Mandibular canal course. Computed tomography. Veterinary dentistry. Brachycephalic dogs.

\section{Introdução}

Os procedimentos cirúrgicos na mandíbula dos cães exigem hoje, do médico veterinário cirurgião, o conhecimento exato da localização de estruturas anatômicas como o canal mandibular (CM), a fim de garantir a integridade dos elementos anatômicos durante procedimentos cirúrgicos odontológicos, e assim,
Correspondência para:

Lenin Arturo Villamizar Martinez

Departamento de Cirurgia, Faculdade de Medicina Veterinária e Zootecnia, Universidade de São Paulo

Av. Prof. Dr. Orlando Marques de Paiva, 87, Bloco 7 superior, Cidade

Universitária, São Paulo-SP, Brasil, CEP 05508-000

e-mail: leninvet@usp.br

Recebido: 23/10/2009

Aprovado: 10/06/2010

Agradecimentos: Ao CNPq, pela bolsa de Mestrado 
preservar a funcionalidade e o bem-estar do animal. O conhecimento da localização do canal mandibular tem grande importância devido à possibilidade de causar trauma iatrogênico ao feixe vasculonervoso alveolar inferior, decorrente da realização de procedimentos cirúrgicos como a extração aberta do dente $1^{\mathrm{o}}$ molar ou a pulverização de raízes fraturadas, a colocação de implantes e a cirurgia ortognática ${ }^{1,2,3,4}$.

O canal mandibular é uma passagem tubular onde estão alojados os vasos sanguíneos e o nervo alveolar inferior ${ }^{5,6}$. O canal mandibular nos cães percorre o corpo da mandíbula na sua porção ventral, desde o forame mandibular localizado na região medial do ramo da mandíbula, ventral à inserção do músculo temporal ${ }^{7}$, até os forames mentonianos caudal, medial e rostral, localizados na superfície vestibular na região rostral da mandíbula, junto aos ápices das raízes dos dentes $1^{\circ}$ e $3^{\circ}$ pré-molares e $1^{\circ}$ ou $2^{\circ}$ dente incisivo respectivamente $\mathrm{e}^{7,8,9,10,11}$.

O diagnóstico por imagem tem tido muita importância nas últimas décadas devido à necessidade de se obter um diagnóstico mais preciso e em menor tempo. Assim, a tomografia computadorizada (TC) tem sido utilizada na Medicina Veterinária como ferramenta de diagnóstico de afecções que comprometem diferentes tecidos do corpo animal, contribuindo sobremaneira com a qualidade do diagnóstico ${ }^{12}$. A tomografia computadorizada emprega os raios $\mathrm{X}$ para formar uma imagem num computador. Esta imagem corresponde a um corte que pode ser realizado em diferentes planos de um objeto sem sobreposição das estruturas internas do mesmo; estas fatias podem ter diferentes espessuras: 0.5, 1, 2, 5 e $10 \mathrm{~mm}^{13,14}$. A tomografia computadorizada pode captar diferenças entre as densidades dos tecidos da ordem de $0,05 \%$, sendo que as radiografias captam diferenças de $0,5 \%$; esta característica melhora a resolução da imagem, permitindo assim detectar alterações nos tecidos que não apareceriam nas radiografias ${ }^{12,15,16}$.
No homem, em áreas como a implantologia e a cirurgia ortognática, o conhecimento exato da localização do canal mandibular é imprescindível ${ }^{17,18}$. Sabe-se amplamente que uma lesão a este feixe vasculonervoso pode produzir desde dor aguda e parestesia transitória pós-cirúrgica durante vários dias no lábio e pele ipsolateral à injúria, até parestesia constante e diminuição da sensibilidade ou contrariamente dor constante ${ }^{19,20}$.

Sabe-se que em mandíbulas normais, no homem, o canal mandibular encontra-se localizado em contato ou muito próximo à cortical lingual do corpo da mandíbula, desde o seu começo no forame mandibular até o forame mentoniano, onde o canal mandibular muda o seu curso rapidamente em direção ao forame mentoniano na superfície vestibular. Ao mesmo tempo em que o canal vai-se tornando vestibular na região do forame mentoniano, este vai incrementando a distância em relação à cortical ventral do corpo da mandíbula; em outras palavras, o canal vai ascendendo progressivamente até o forame mentoniano ${ }^{17,21}$. Em pesquisa realizada por Villamizar et al. ${ }^{22}$ em cães braquicefálicos, o canal mandibular desceu suavemente desde a região do forame mandibular, até a região do dente 1ำ molar onde alcança a sua máxima profundidade. O CM localizou-se a distâncias similares entre as faces vestibular e lingual do corpo da mandíbula antes de terminar na face vestibular no forame mentoniano medial.

\section{Material e Método}

Foram utilizadas dez cabeças de cadáveres de cães adultos mesaticefálicos (segundo o índice cefálico de Evans e Christensen ${ }^{6}$ ), sem padrão racial definido (SRD), que vieram a óbito no HOVET-FMVZ-USP. A metodologia empregada no desenvolvimento deste trabalho foi julgada e aprovada pela Comissão de Bioética da FMVZ-USP. 
O periodonto das mandíbulas foi avaliado com ajuda de sonda exploradora e sonda milimetrada. Unicamente as mandíbulas com periodonto saudável foram utilizadas nesta pesquisa enquanto que as mandíbulas que apresentaram doença periodontal moderada ou grave foram descartadas devido à possibilidade de apresentarem perda de osso alveolar e subsequente diminuição do nível normal da margem alveolar da mandíbula.

O exame tomográfico foi realizado no Serviço de Diagnóstico por Imagem do Hospital Veterinário da Faculdade de Medicina Veterinária da Universidade de São Paulo. Os exames tomográficos da mandíbula foram obtidos com ajuda do tomógrafo de terceira geração CT MAX $640^{\circ}$ (General Electric); as imagens foram fotografadas com câmara multiformato MFC640 ${ }^{\circledR}$ (General Electric) em filmes Kodak Ektanscan $\mathrm{M}$ de $35 \mathrm{x} 43 \mathrm{~cm}$ e posteriormente reveladas e fixadas em processadora automática RPX- OMAT (Eastman Kodak Company). A técnica empregada no tomógrafo foi de $120 \mathrm{kV}, 22 \mathrm{~mA}$ e 4,8 segundos de tempo de aquisição de dados com reconstrução para tecido ósseo; a espessura do corte foi de $2 \mathrm{~mm}$.

Foi realizado um scout (radiografia digital) lateral inicial para visibilizar as estruturas anatômicas que serviriam como referência para os cortes transversais (Figura 1). Estes pontos de referência foram: o forame mandibular, as raízes dos dentes molares, pré-molares e o forame mentoniano medial. As cabeças foram posicionadas de tal forma que a borda ventral da mandíbula e consequentemente o canal mandibular ficassem no plano horizontal, paralelos à mesa, assim, os cortes transversais foram realizados o mais perpendicularmente possível com relação ao canal mandibular, evitando assim distorções de sua imagem e incrementando o detalhe da imagem do canal mandibular.

Para determinar a relação anatômica do canal mandibular no interior do corpo da mandíbula foram realizadas diferentes medidas nos tomogramas transversais; estas medições foram feitas desde o limite externo do canal mandibular até as diferentes superfícies (no plano horizontal e vertical) do corpo da mandí-

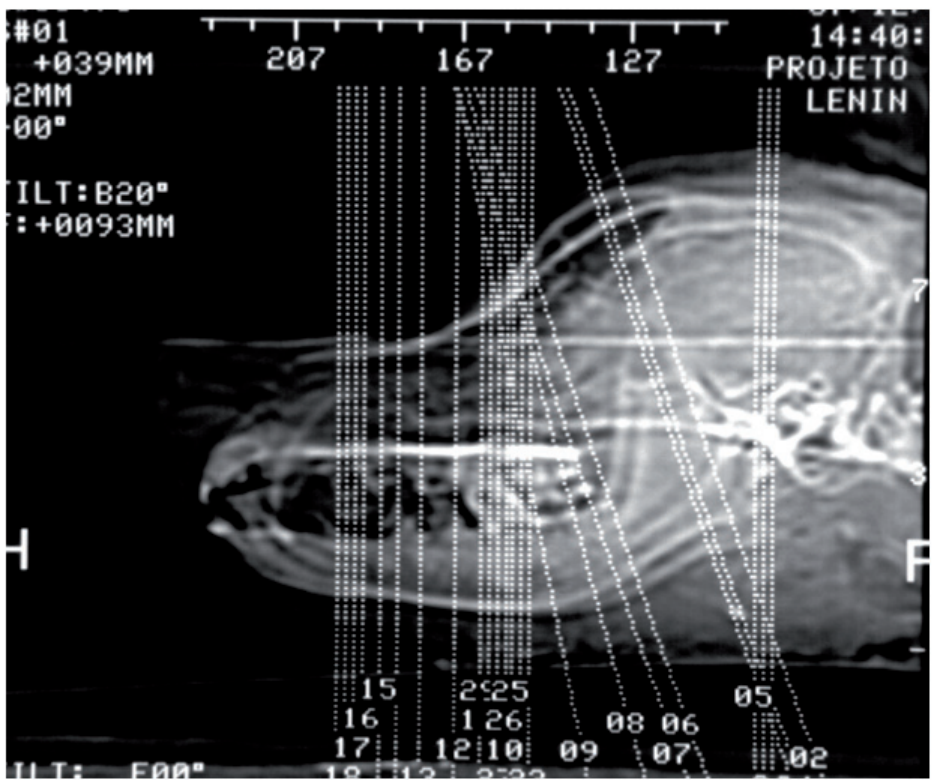

Figura 1 - Radiografia digital (Scout), imagem lateral da cabeça de um cão mesaticefálico; as linhas brancas numeradas representam o local onde os cortes transversais foram realizados 
bula: altura e largura da mandíbula, distância entre o canal mandibular e as faces vestibular, lingual, ventral e a margem alveolar (profundidade do canal) do corpo da mandíbula, (Figura 2). As mensurações foram submetidas à análise estatística descritiva com ajuda do programa $\mathrm{R}^{\oplus}$ versão 2.4.1. (programa desenvolvido para análise estatística e gráfica). Este programa permitiu obter a média, o desvio padrão e o intervalo (medida maior e menor) correspondente a cada mensuração realizada nos cães.

\section{Resultados}

A mandíbula foi dividida em quatro regiões para sintetizar as mensurações realizadas: região do forame mandibular (FM), região molar (entre os dentes molares $1^{\circ}$ e $3^{\circ}$ ), região pré-molar (entre os dentes pré-molares $2^{\circ}$ e $\left.4^{\circ}\right)$ e região do forame mentoniano medial. Os valores relacionados na tabela 1 correspondem à média e desvio padrão das mensurações realizadas em cada região da mandíbula.
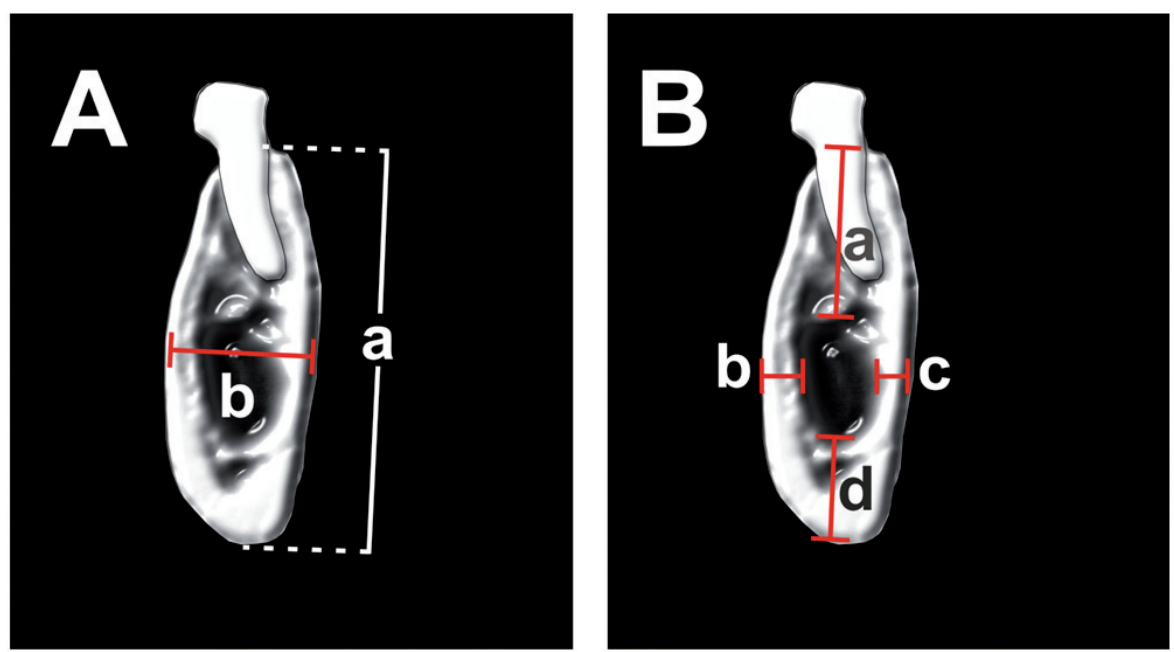

Figura 2 - Esquemas de cortes transversais do dente $2^{\circ}$ molar mandibular; diferentes medidas foram realizadas no corpo da mandíbula: (A) (a) altura e (b) largura; (B) (a) profundidade do CM, (b) distância entre o CM e a face vestibular, (c) distância entre o CM e a face lingual e (d) distância entre o $\mathrm{CM}$ e a face ventral

Tabela 1 - Média e desvio padrão das mensurações realizadas em cada região da mandíbula em cães mesaticefálicos, considerando como referência os forames (mandibular e mentoniano medial) e as raízes dentárias dos dentes molares e pré-molares

\begin{tabular}{|c|c|c|c|c|c|c|}
\hline $\begin{array}{c}\text { Ponto de } \\
\text { referência na } \\
\text { mandibula }\end{array}$ & $\begin{array}{c}\text { Altura da } \\
\text { mandíbula } \\
(\mathrm{mm})\end{array}$ & $\begin{array}{c}\text { Largura da } \\
\text { mandíbula } \\
(\mathrm{mm})\end{array}$ & $\begin{array}{c}\text { Distância entre } \\
\text { o CM e a face } \\
\text { vestibular (mm) }\end{array}$ & $\begin{array}{c}\text { Distância entre } \\
\text { o CM e a face } \\
\text { lingual (mm) }\end{array}$ & $\begin{array}{c}\text { Distância entre } \\
\text { o CM e a face } \\
\text { ventral }(\mathrm{mm})\end{array}$ & $\begin{array}{c}\text { Profundidade } \\
\text { do CM } \\
\text { (mm) }\end{array}$ \\
\hline Forame mandibular & - & $3,16 \pm 0,53$ & $1,02 \pm 0,15$ & 0 & $9,09 \pm 1,42$ & - \\
\hline $3^{\circ}$ Molar & $21,87 \pm 2,90$ & $7,55 \pm 1,11$ & $2,43 \pm 0,68$ & $1,54 \pm 0,25$ & $5,74 \pm 1,07$ & $9,86 \pm 1,58$ \\
\hline RD $2^{\circ}$ Molar & $20,88 \pm 2,57$ & $7,94 \pm 0,66$ & $2,53 \pm 0,38$ & $1,71 \pm 0,26$ & $5,17 \pm 1,04$ & $9,45 \pm 1,43$ \\
\hline RM $2^{\circ}$ Molar & $20,44 \pm 2,90$ & $8,07 \pm 0,82$ & $2,43 \pm 0,36$ & $1,77 \pm 0,56$ & $4,28 \pm 0,98$ & $10,4 \pm 1,85$ \\
\hline $\mathrm{RD} 1^{\circ}$ Molar & $20,02 \pm 3,07$ & $8,38 \pm 0,87$ & $2,27 \pm 0,25$ & $1,81 \pm 0,49$ & $3,31 \pm 0,87$ & $11,4 \pm 1,61$ \\
\hline RM $1^{\circ}$ Molar & $21,10 \pm 3,16$ & $8,67 \pm 1,19$ & $2,02 \pm 0,36$ & $2,13 \pm 0,19$ & $2,54 \pm 0,40$ & $13,62 \pm 2,22$ \\
\hline RD $4^{\circ}$ Pré-molar & $20,06 \pm 3,48$ & $8,41 \pm 0,87$ & $2,35 \pm 0,29$ & $1,97 \pm 0,29$ & $2,37 \pm 0,21$ & $12,16 \pm 1,93$ \\
\hline RM $4^{\circ}$ Pré-molar & $19,51 \pm 3,72$ & $8,42 \pm 0,87$ & $2,26 \pm 0,28$ & $2,05 \pm 0,20$ & $2,31 \pm 0,34$ & $11,57 \pm 2,10$ \\
\hline RD $3^{\circ}$ Pré-molar & $18,42 \pm 3,22$ & $8,28 \pm 0,89$ & $2,2 \pm 0,32$ & $2,2 \pm 0,20$ & $2,29 \pm 0,25$ & $10,77 \pm 1,70$ \\
\hline RM $3^{\circ}$ Pré-molar & $17,75 \pm 3,16$ & $8,52 \pm 0,84$ & $2,04 \pm 0,36$ & $2,59 \pm 0,73$ & $2,4 \pm 0,82$ & $10,44 \pm 2,21$ \\
\hline RD $2^{\circ}$ Pré-molar & $17,76 \pm 2,92$ & $8,89 \pm 1,00$ & $1,71 \pm 0,48$ & $4,13 \pm 1,06$ & $4,53 \pm 1,44$ & $9,56 \pm 1,76$ \\
\hline RM $2^{\circ}$ Pré-molar & $18,08 \pm 2,96$ & $8,99 \pm 1,10$ & $0,72 \pm 0,68$ & $5,69 \pm 1,48$ & $6,18 \pm 1,67$ & $9,07 \pm 1,78$ \\
\hline Forame Mentoniano & $18,13 \pm 2,87$ & $8,7 \pm 1,63$ & 0 & $6,29 \pm 1,17$ & $7,17 \pm 1,68$ & $8,75 \pm 1,47$ \\
\hline
\end{tabular}

(RM) raiz mesial; (RD) raiz distal; (-) não mensurado 
O canal mandibular esteve localizado no aspecto lingual do corpo da mandíbula, desde o forame mandibular até o dente $1^{\circ}$ molar. A partir deste ponto, localizou-se a distância similar, entre a face lingual e vestibular, até a região do $3^{\circ}$ pré-molar e após este ponto dirigiu-se para a face vestibular, afastando-se da face lingual antes do seu fim no forame mentoniano medial. Em relação à margem alveolar (profundidade), o CM descendeu progressivamente desde o forame mandibular até alcançar a sua máxima profundidade, no dente $1^{\circ}$ molar, e logo ascendeu, afastando-se da cortical ventral para terminar, assim, no forame mentoniano medial (Figuras $3,4,5$ e 6$)$.
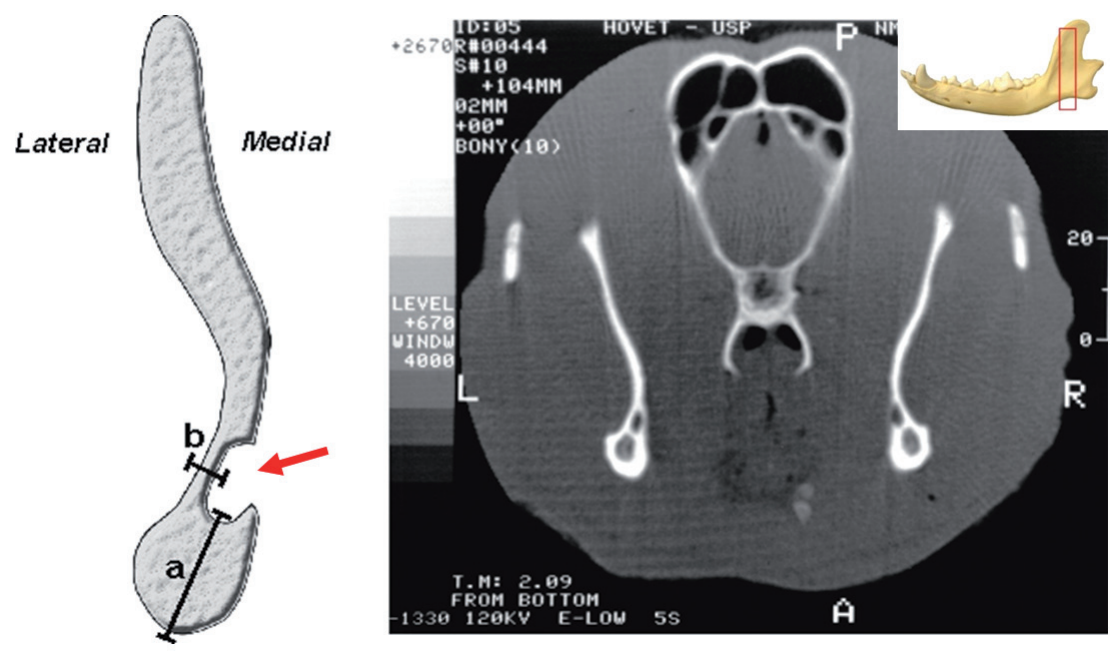

Figura 3 - A figura mostra a área do forame mandibular amplificada a partir de um tomograma. O forame mandibular aparece na face medial do ângulo da mandíbula como uma pequena depressão no terço distal do processo coronoide (seta), separado da face ventral por osso medular (a) e lateralmente da fossa massetérica apenas por uma fina lâmina de osso (b)
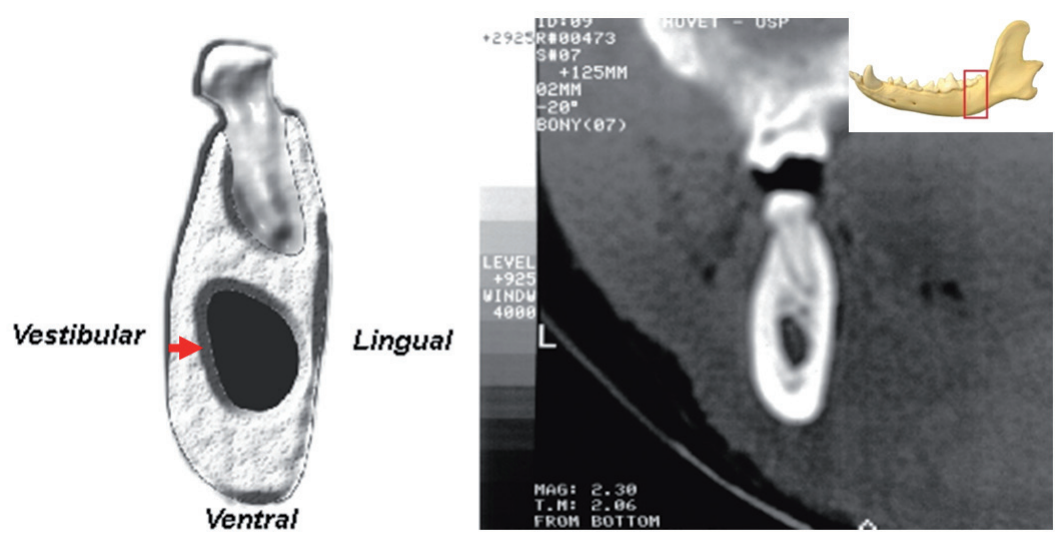

Figura 4 - Na região dos dentes $2^{\circ}$ e $3^{\circ}$ molar, o CM foi localizado ventral às raízes dentárias, discretamente mais próximo da face lingual do que da face vestibular do corpo da mandíbula 

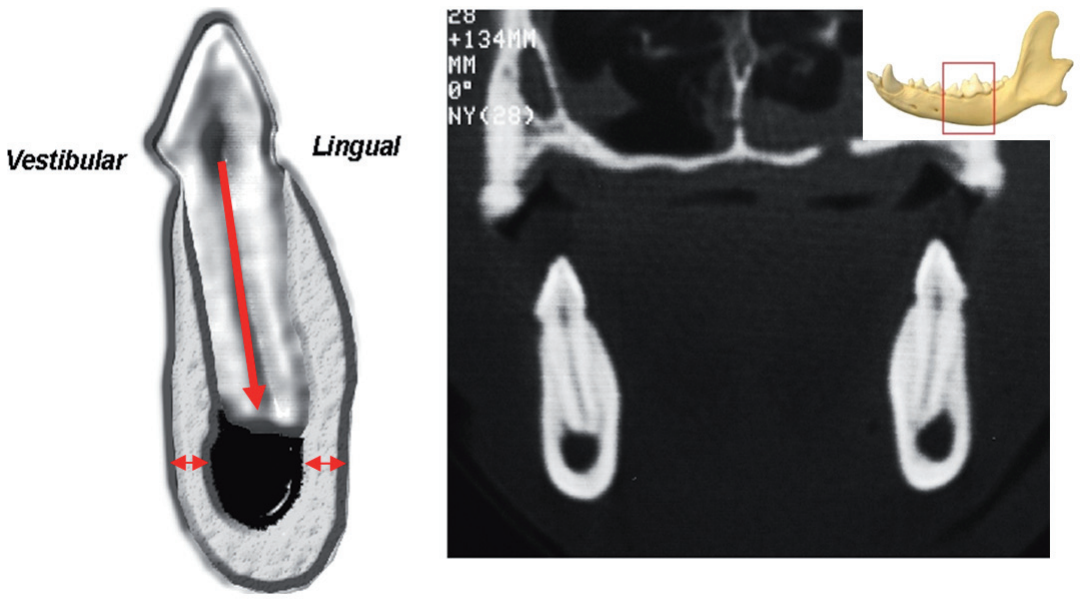

Figura 5 - Na região do dente $1^{\circ}$ molar, o CM foi localizado na porção ventral do corpo da mandíbula, separado unicamente pela cortical da face lingual e vestibular, alcançando sua máxima profundidade
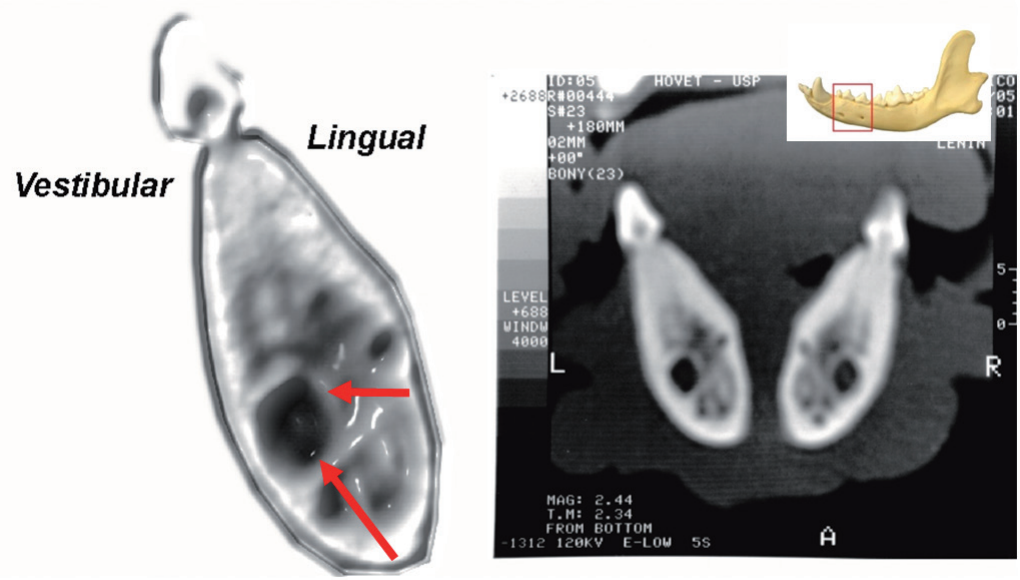

Figura 6 - Na região rostral da mandíbula correspondente ao dente $2^{\mathrm{o}}$ pré-molar, o $\mathrm{CM}$ incrementou a distância com relação à face lingual e ventral (setas) antes do seu final no forame mentoniano medial

As raízes dentárias localizaram-se geralmente no aspecto dorsal do canal mandibular e raramente apresentaram protrusão para o interior do mesmo.

\section{Discussão}

Com base nos resultados obtidos neste trabalho, sugere-se que o trajeto do canal mandibular nos cães mesaticefálicos é similar ao trajeto descrito por Villa- mizar et al. ${ }^{22}$ nos cães braquicefálicos e que difere moderadamente do trajeto do canal mandibular no Homem, no qual o mesmo foi localizado através do corpo da mandíbula em contato ou muito próximo à face lingual em toda a sua extensão, como foi descrito na literatura por Gowgiel ${ }^{17}$ e Suazo et al. ${ }^{21}$. Procedimentos cirúrgicos em cães, como a colocação de implantes, devem se restringir à região dorsal do CM, 
devido a pouca quantidade de osso que separa o canal mandibular das faces vestibular e lingual, diferentemente do que ocorre no Homem, no qual, pelo fato de que o CM ser localizado mais próximo da face lingual do corpo da mandíbula, a colocação de implantes orientados para sua face vestibular é possível, como foi relatado por Gowgiel ${ }^{17}$.

A manipulação de alavancas para exodontia de dentes molares e pré-molares da mandíbula deve ser realizada com precaução, controlando-se a força vertical aplicada com alavancas pelo cirurgião, devido à possibilidade de se atingir o CM no intento de luxar o dente. Por outro lado, a pulverização de fragmentos de raiz com canetas de alta rotação e pontas diamantadas deve ser evitada, pela alta possibilidade de alcançar o $\mathrm{CM}$ ou pela possibilidade de se introduzir fragmentos de raiz ou material contaminado dentro do canal, que posteriormente podem produzir desconforto e dor ao paciente, concordando com o descrito por Reiter ${ }^{4}$, Marreta $^{2}$, Kubilius et al. ${ }^{20}$ e Kraut e Chahal ${ }^{19}$.

A tomografia computadorizada demonstrou-se uma ferramenta de grande valor para avaliação morfológica e volumétrica do componente ósseo da mandíbula, permitindo estabelecer a relação do canal mandibular com as diferentes faces da mandíbula. A capacidade de obter imagens sem sobreposição de estruturas,

\section{Referências}

1. CARVALHO, V. G. Cirurgia ortognática em cães: técnica intraoral de osteotomia sagital da mandíbula para correção de prognatismo e retrognatismo. Estudo em cadáveres. 2008. 164 p. Tese (Doutorado em Clínica Cirúrgica Veterinária) Faculdade de Medicina Veterinária e Zootecnia, Universidade de São Paulo, São Paulo, 2008.

2. MARRETA, S. M. Surgical extraction of the first molar tooth in the dog. Journal of Veterinary Dentistry, v. 19, n. 1, p. 4650, 2002.

3. RAPLEY, J. W.; H.SWAN, R.; CRONIN, R. J.; MATHEY, W. S. The use of dental implants for the rehabilitation of the military working dog: a case report. Journal of the American Animal Hospital Association, v. 27, n. 6, p. 641$645,1991$.

4. REITER, A. M. Dental surgical procedures. In: TUTT, C.; DEPROSE, J.; CROSSLEY, D. (Ed.). BSAVA manual of canine and feline dentistry. 3. ed. Gloucester: British Small Animall Animal Veterinary Association, 2007. p. 178-195. junto à possibilidade de empregar as ferramentas próprias do programa do tomógrafo para a realização de medidas, pode oferecer ao médico veterinário uma série de informações importantes a serem usadas na avaliação pré-cirúrgica de procedimentos odontológicos veterinários.

As atuais necessidades da cirurgia maxilofacial em cães reafirmam a tomografia computadorizada como uma ferramenta de grande valor diagnóstico, na avaliação pré e pós-cirúrgica do paciente. $\mathrm{O}$ emprego de placas e miniplacas metálicas e parafusos na cirurgia ortopédica ou ortognática, a colocação de implantes dentários ou a realização de procedimentos rotineiros como a exodontia na região da mandíbula requerem planejamento e conhecimento das estruturas anatômicas envolvidas nos procedimentos, para não comprometer a integridade de estruturas nervosas ou vasculares como as que percorrem dentro do canal mandibular.

Com base no exposto neste trabalho, e devido à escassez de trabalhos que envolvam o uso da tomografia computadorizada para fins odontológicos na Medicina Veterinária, recomenda-se a realização de mais estudos que envolvam esta técnica, visando detalhar as características anatômicas da cavidade oral de diferentes raças de cães.

5. BLOOD, D. C.; STUDDERT, V. P.; GAY, C. C. Saunders comprehensive veterinary dictionary. 3 . ed. Philadelphia: Saunders Elsevier, 2007. p. 285

6. EVANS, H. E.; CHRISTENSEN, G. C. The heart and arteries. In: EVANS, H. E.; CHRISTENSEN, G. C. Miller's anatomy of the dog. $2^{\text {nd }}$ ed. Philadelphia: W.B. Saunders Company, 1979. p. 665-666.

7. EVANS, H. E.; CHRISTENSEN, G. C. The skeleton. In: EVANS, H. E.; CHRISTENSEN, G. C. Miller's anatomy of the dog. $2^{\text {nd }}$ ed. Philadelphia: W.B. Saunders Company, 1979. p. $146-148$.

8. BELLOWS, J. E.; DUMAIS, Y.; GIOSO, M. A.; REITER, A. M.; VERSTRAETE, F. J. Foundations: clarification of veterinary dental nomenclature. Journal of Veterinary Dentistry, v. 22, n. 4 , p. 272-279, 2005

9. EUBANKS, D. L. Oral soft tissue anatomy in the dog and cat. Journal of veterinary Dentistry, v. 24, n. 2, p. 126-129, 2007. 
10.GRACIS, M. Orodental anatomy and physiology. In: TUTT, C.; DEEPROSE, J.; CROSSLEY, D. A. (Ed.). BSAVA manual of canine and feline dentistry. 3. ed. Gloucester: British Small Animal Veterinary Association, 2007. p. 9-21.

11.HARVEY, C. E.; EMILY, P. P. Funtion, formation, and anatomy of oral structures in carnivores. In: HARVEY, C. E.; EMILY, P. P. Small animal dentistry. St. Louis: Mosby, 1993a. p. 1-18.

12.FARFALLINI, D. Tomogafia computarizada. In: PELLEGRINO, F.; SURANITI, A. El libro de neurologia para la práctica clínica. Buenos Aires: Intermedica, 2003. p. 475-481.

13. ASSHEUER, J.; SAGER, M. Principles of imaging techniques. In: ASSHEUER, J.; SAGER, M. MRI and CT atlas of the dog. Berlin: Blackwell Science, 1997. p. 449-461.

14.FEENEY, D. A.; FLETCHER, T. F.; HARDY, R. M. Multiplanar imaging. In: FEENEY, D. A.; FLETCHER, T. F.; HARDY, R. $\mathrm{M}$. Atlas of correlative imaging anatomy of the normal dog. Philadelphia: W.B. Saunders Company, 1991. p. 335-352.

15.REVENAUGH, A. F. Computed tomography and magnetic resonance imaging in veterinary dentistry and oral surgery diagnostics. In: DEFORGE, D. H.; COLMERY, B. H. (Ed.). An atlas of veterinary dental radiology. 1. ed. Ames: Iowa State University Press, 2000. p. 261-263.

16. TIDWELL, A. S. Principles of computed tomography and magnetic resonance imaging. In: THRALL, D. E. Textbook of veterinary diagnostic radiology. 5. ed. St. Louis: Saunders Elsevier, 2007. p. 50-56.

17. GOWGIEL, J. M. The position and course of the mandibular canal. Journal of Oral Implantology, v. 18, n. 4, p. 383-385, 1992.

18.SERHAL, C. B.; STEENBERGHE, D. V.; QUIRYNEN, M.; JACOBS, R. Localization of the mandibular canal using conventional spiral tomography: a human cadaver study. Clinical Oral Implants Research, v. 12, n. 3, p. 230-236, 2001.

19.KRAUT, R. A.; CHAHAL, O. Magagement of patients with trigeminal nerve injuries after mandibular implant placement. Journal of the American Dental Association, v. 133, n. 10, p. 1351-1354, 2002.

20.KUBILIUS, R.; SABALYS, G.; JUODZBALYS, G.; GEDRIMAS, $V$. Traumatic damage to the inferior alveolar nerve sustained in course of dental implantation. Possibility of prevention. Stomatologija, Baltic Dental and Maxillofacial Journal, v. 6, n. 4, p. 106-110, 2004.

21.SUAZO, G.; MORALES, H.; CANTÍN, L.; ZAVANDO, M. Aspectos biométricos del canal mandibular. International Journal of Morphology, v. 25, n. 4, p. 811-816, 2007.

22.VILlAMIZAR, L. A.; GIOSO, M. A.; VILlEGAS, C. M.; PINTO, A. C. B. Localization of the mandibular canal in brachycephalic dogs using computed tomography. Journal of Veterinary Dentistry, v. 26, n. 3, p. 156-163, 2009. 